\title{
So entsteht künftig das Honorarbudget einer Praxis
}

- Nach der Trennung der um die Honorare für die Richtlinienpsychotherapie, die Laborleistungen und die im organisierten Notdienst erbrachten Leistungen bereinigten Gesamtvergütung werden $a b$ dem 1.7.2010 - im Vergleich zur bisherigen Regelung - nur noch wenige verbleibende Leistungen außerhalb der Regelleistungsvolumina (RLV) vergütet. Relevant für den hausärztlichen Bereich sind hier allenfalls noch die Kostenpauschalen und dort die Wegepauschalen für Hausbesuche. Alle übrigen Leistungen unterliegen einer Mengenbegrenzung im Rahmen des RLV oder - und das ist neu eines Qualitätszuschlages (OZZV).

Die Berechnung der qualifikationsgebundenen Zusatzvolumen (QZZ) erfolgt arztgruppenspezifisch auf der Basis der abgerechneten Punktzahlen des Jahres 2008 für jedes QZZV einzeln. Jeder Arztgruppe steht dafür ein bestimmtes Verteilungsvolumen zur Verfügung, aus dem die unterschiedlichen Zusatzvolumen bedient werden.

Zur Ermittlung der qualifikationsgebundenen Zusatzvolumen hat der Bewertungsausschuss drei Rechenwege vorgegeben, die in den einzelnen Kassenärztlichen Vereinigungen mit den regionalen Krankenkassen wahlweise vereinbart werden können.

Bei der bekanntesten Methode der Berechnung nach Behandlungsfall erhält jeder Arzt einer Arztgruppe, der Anspruch auf das jeweilige Zusatzvolumen hat, einen Zuschlag pro RLV-Fall. Zur Berechnung wird die Fallzahl des Arztes im Vorjahresquartal mit dem Fallwert der Arztgruppe für das jeweilige qualitätsbezogene Zusatzvolumen multipliziert. Der QZV-Fallwert der Arztgruppe errechnet sich dabei aus dem für das jeweilige Zusatzvolumen bereitstehenden Verteilungsvolumen geteilt durch die Fallzahl der Ärzte, die Anspruch auf das QZV haben.

Hatte ein Arzt z.B. im Vorjahresquartal 1000 RLV-Fälle und davon 150 dringende Hausbesuche abgerechnet, erhält er für alle 1000 Fälle einen Zuschlag in Höhe des errechneten OZV-Fallwertes der Fachgruppe. Der QZZV-Fallwert der Arztgruppe dürfte bei dieser Berechnungsmethode jedoch relativ gering ausfallen, es fließen aber in die Berechnung des Zusatzvolumens alle Fälle des Arztes ein. Sein eigenes Budget wird dadurch höher, der RLVFallwert der Gruppe niedriger ausfallen. Diese Auswirkung wird durch den Bezug auf den Leistungsfall als Berechnungsgrundlage reduziert. Bei dieser Variante erhält jeder Arzt, der Anspruch auf das jeweilige Zusatzvolumen hat, nur einen Zuschlag pro Leistungsfall. Dieser liegt vor, wenn im Behandlungsfall mindestens eine Leistung des entsprechenden QZV abgerechnet worden ist. Zur Berechnung des Zuschlags wird die Leistungsfallzahl des Arztes im Vorjahresquartal mit dem Fallwert der Arztgruppe für das jeweilige QZV multipliziert. Der Fallwert errechnet

\section{MMW Kommentar}

Ziel der Reform zum 1.7.2010 ist eine wesentlich bessere Ausstattung des Honorarvolumens, das für die RLV zur Verfügung steht, und damit eine Anhebung des RLV-Fallwertes. Soll dies gelingen, dürfte auch nur die Berechnungsmethode für die QZV mit der geringsten RLV-Belastung gewählt werden. Dies garantiert nur der arztbezogene Rechenweg. Die Fallzahl spielt hier keine Rolle, eine Fallzahlausweitung folgerichtig auch nicht. Hier wird das für das jeweilige QZV zur Verfügung stehende Honorarvolumen der Arztgruppe durch die Anzahl der Ärzte der Arztgruppe geteilt, die Anspruch auf das jeweilige QZV haben. Bei sinkender Arztzahl in der Gruppe steigt

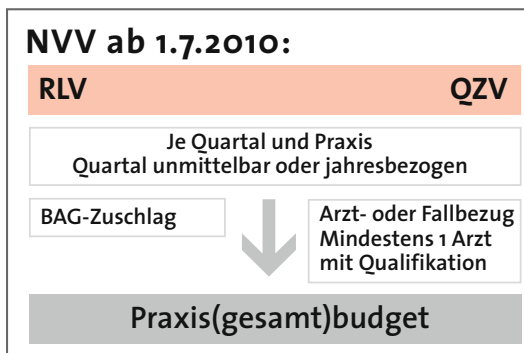

Abb.: Ab dem 1.7.2010 hat die Praxis ein Gesamthonorarbudget, das sich aus RLV- und bisherigen Vorableistungen zusammensetzt. Bei der Abrechnung der Leistungen kann allerdings das resultierende Gesamtbudget insgesamt verwendet werden.

sich aus dem für das Zusatzvolumen vorhandenen Verteilungsvolumen geteilt durch die Leistungsfallzahl der Ärzte der Arztgruppe. Der Arzt, der im Vorjahresquartal 1000 RLV-Fälle und dabei 150 dringende Hausbesuche abgerechnet hatte, erhält hier nur für 150 Leistungsfälle (Hausbesuchsfälle) einen Zuschlag in Höhe des OZZ-Leistungsfallwertes. dann auch das zur Verfügung stehende Honorarvolumen. Jeder Arzt hat dann das gleiche QZV, unabhängig davon, wie viele Fälle und wie viele dringende Hausbesuche er im Vorjahresquartal erbracht hat.

Das klingt zunächst negativ, bedeutet im konkreten Fall aber, dass über den hier zu erwartenden höchsten Anstieg des RLV-Fallwertes auch die normalen Hausbesuche besser vergütet werden. Aus betriebswirtschaftlicher Sicht fällt die Methodenwahl deshalb leicht. Welche Berechnung sich letztendlich unter berufspolitischen Gesichtspunkten durchsetzt, wird in den kommenden Wochen und Monaten hingegen Gegenstand heftiger Diskussionen sein. 